\section{ORIGINAL RESEARCH}

R.N. Walker

I.J. Alexander

J.A. Sartorius

C.A. Woomert

\title{
Anthropometric Measurements: Effect of CT Depth of Pretracheal Soft Tissue on Tracheotomy Tube Selection
}

BACKGROUND AND PURPOSE: Tracheotomy is a commonly performed procedure; however, in the obese, it can be associated with a high morbidity and mortality, partially due to accidental decannulation. We hypothesize that a simple and rapid measurement of the DPST on CT will accurately predict those patients in need of an extended-length tracheotomy tube.

MATERIALS AND METHODS: A retrospective review of the electronic health record and available CT imaging of the neck was performed for all patients who underwent tracheotomy at a tertiary care center. Measurement of the DPST was performed and compared with the recorded tracheotomy tube used at surgery.

RESULTS: Five hundred twenty-two adult patients underwent an initial tracheotomy procedure, of whom 293 met the inclusion criteria for the study. A statistically significant correlation between the DPST and the need for an extended-length tracheotomy tube was seen. A discriminatory soft-tissue depth indicating the need for an extended-length tracheotomy tube was calculated.

CONCLUSIONS: CT measurement of the anterior soft-tissue depth is a simple tool that appears to provide a discriminatory threshold for the need for an extended-length tracheotomy tube. Further validation of this model through prospective application is needed. Although routine use of CT as a pretracheotomy evaluation is not advocated, the anatomic information is often available through prior imaging and has the potential to decrease the incidence of tracheotomy tube dislodgement in the obese and subsequent morbidity.

ABBREVIATIONS: $\mathrm{BMI}=$ body mass index; $\mathrm{Cl}=$ confidence interval; DPST $=$ depth of pretracheal soft tissue; ENT = ear, nose, and throat; $O R=$ odds ratio

$\mathbf{T}$ racheotomy has increased in popularity as a means of longterm ventilation for patients. Today, it is one of the most commonly performed surgical procedures and is generally tolerated well. Several complications of tracheotomy are wellestablished in the literature. One of the most serious initial complications of tracheotomy tube placement is that of tube dislodgement, which is reported to occur in approximately $1.5 \%$ of procedures. ${ }^{1}$ Rates of complications in obese patients, however, are higher in several series. ${ }^{2,3}$

Tube displacement results in airway compromise, often with resultant long-term morbidity or mortality from hypoxia. ${ }^{1,4}$ Several types of tracheotomy tube classes are available for procedures, designed to fit specific patient populations. Most patients are fitted with a standard-length tube, which come in a variety of internal diameters. Proximal extendedlength tubes are less commonly used but become necessary in patients with abundant anterior soft tissue. ${ }^{4,5}$ The choice of tube length and type is often guided by the surgeon's experi-

Received May 25, 2011; accepted after revision June 29

From the Departments of Radiology (R.N.W.) and Otolaryngology (I.J.A.), Center for Health Research (J.A.S.), and Department of Radiology (C.A.W.), Geisinger Medical Center, Danville, Pennsylvania. Dr Walker is currently with Irvington Radiologists, Indianapolis, Indiana. Dr Alexander is currently with ENT of Los Alamos, Los Alamos, New Mexico.

Paper previously presented at: Annual Meeting of the American Society of Neuroradiology, May 16-21, 2009; Vancouver, British Columbia, Canada; and Annual Meeting of the American Society of Head and Neck Radiology, October 7-11, 2009; New Orleans, Louisiana.

Please address correspondence to Robert N. Walker, MD, MPH, Irvington Radiologists, 7340 Shadeland Station, Suite 200, Indianapolis, IN 46256-2150; e-mail: robert.n.walker@ gmail.com

http://dx.doi.org/10.3174/ajnr.A2823 ence, a qualitative evaluation of patient body habitus, and tube fit during the procedure.

Tube dislodgement typically occurs within a few days of the procedure. The causes of tube dislodgement are multifactorial and include tube length, neck thickness, postoperative swelling, and poor securing of the tube. ${ }^{1}$ Neck thickness is often related to obesity, and obesity rates in the United States are increasing.

The purpose of this study was to determine whether an independent metric, the depth of soft tissue over the tracheotomy site as measured on CT, can be used as a guideline for the appropriate length of the tracheotomy tube needed for placement. If a quantitative objective metric to identify those patients who may benefit from a proximal extended-length tube could be determined, it may positively affect tube-dislodgement rates.

\section{Materials and Methods}

Following an institutional review board-approved process, we performed a retrospective review of the electronic medical record to identify adult patients who underwent tracheotomy tube placement at a single tertiary care institution during a 5-year period from 2003 through 2008. Patients who did not have a CT scan covering the level of the first and second tracheal rings within 1 month before surgery were excluded from the study. Although the study was initially intended to evaluate $\mathrm{CT}$ of the neck only, it was found during record review that occasionally a $\mathrm{CT}$ of the chest was inadvertently extended cranially and covered the appropriate anatomic region of concern. CT of the chest should not, however, be routinely extended to include the thyroid gland or tissues superior to the thyroid due to the concern for 
radiation exposure to the thyroid gland. Nonetheless, this information was included in our review and analyzed separately as described below due to differences in patient positioning.

CT was performed on multiple different CT units available in the department during the range of time included in the retrospective review: Picker PQ 5000 (single-section spiral CT; Picker International, Cleveland, Ohio), LightSpeed 16 (16-section multidetector CT; GE Healthcare, Milwaukee, Wisconsin), LightSpeed VCT (64section multidetector CT, GE Healthcare), and Discovery ST (16section multidetector PET/CT, GE Healthcare). Due to the variety of CT units in use in the department during the course of the review, no consistent protocol could be applied to the reviewed studies.

The CT scans and electronic health records of the patients were reviewed. Measurement of the DPST at the level of the first and second tracheal rings was performed and correlated to the choice of tracheotomy tube at surgery. Although tracheotomy can be performed at a variety of levels in the trachea, the first and second tracheal rings were chosen due to their relative ease of identification as a fixed level measurement to provide comparison. In addition, the arm position (typically arms at the sides for neck CT and arms elevated above the head for chest CT) was recorded because it was thought that arm position may have an effect on the DPST measurement. The postoperative course was reviewed to evaluate complications.

Preliminary measurement data were collected from a random selection of 19 patients who underwent neck CT and did not have a tracheotomy procedure recorded in the medical record. The samplesize calculations were performed by using an $\alpha$ of .05 and a power of $80 \%$ for an anticipated 2 -sided test. Sample size by using the average soft-tissue measurement in the preliminary group $(1.5 \mathrm{~cm})$ indicated that 16 patients in each group would be needed to test a differentiation of at least $0.7 \mathrm{~cm}$.

Patients were grouped according to several variables: use of proximal extended-length tracheotomy tube, surgeon type (ENT or general), BMI, and reason for tracheotomy. BMI was divided into 4 categories: $<25=$ healthy, $25-29=$ overweight, $30+=$ obese, and unknown. The distribution of all variables was evaluated. Bivariate comparisons were checked between the outcome and all possible predictors (Fisher exact tests were used for categoric variables, and 2-sample $t$ tests, for continuous variables). Logistic models were then fit by using the DPST measured by CT for predicting whether an extender was used; then, a logistic model was performed with BMI as the main predictor. Both the final models for BMI (adjusted for ENT) and DPST (adjusted for arm position) were checked for lack of fit by using the Hosmer-Lemeshow test. The 2 models were then compared on predictive power by using the same pseudo- $R^{2}$ measure. All significant covariates were included in the final model. Interaction terms were checked. All analysis was done by using the statistical software SAS 2002-2008 (SAS Institute, Cary, North Carolina), and $P$ values $<$ .05 were considered statistically significant.

\section{Results}

Five hundred twenty-two patients underwent tracheotomy tube placement during the review period. Of these, 293 patients had an appropriate CT and were eligible for analysis (56\%). Nineteen patients $(6.5 \%)$ had a tube placed with an extended proximal length. Patient data are summarized in Tables 1 and 2. Those who required an extended-length tube had significantly higher average BMIs and were more likely to have their procedure performed by an otolaryngologist. In addition, those who required an extended-length tube had a sig-
Table 1: Patient demographics

\begin{tabular}{lccr}
\hline & \multicolumn{3}{c}{ Proximal } \\
& $\begin{array}{c}\text { Standard-Length } \\
\text { Tube }\end{array}$ & $\begin{array}{c}\text { Extended-Length } \\
\text { Tube }\end{array}$ & $P$ \\
& $(n=274)$ & $(n=19)$ & Value \\
\hline \% Male & $66.10 \%$ & $52.60 \%$ & .235 \\
Age (mean) & $56.5 \pm 19$ & $59.7 \pm 13$ & .468 \\
BMI (mean), 65\% known & $28.4 \pm 7$ & $48.9 \pm 14$ & $<.001$ \\
DPST (cm) (mean) & $2.2 \pm 1$ & $6.2 \pm 2$ & $<.001$ \\
$\%$ Associated spine finding & 27.00 & 42.10 & .156 \\
$\%$ Having ENT surgeon & 36.10 & 68.40 & .005 \\
\hline
\end{tabular}

\begin{tabular}{|c|c|c|c|}
\hline & $\begin{array}{c}\text { Standard-Length } \\
\text { Tube } \\
(n=274)\end{array}$ & $\begin{array}{c}\text { Proximal } \\
\text { Extended-Length } \\
\text { Tube } \\
(n=19)\end{array}$ & $\begin{array}{c}P \\
\text { Value }\end{array}$ \\
\hline Reason for tracheotomy & & & .146 \\
\hline$\%$ Acute respiratory failure & 12.00 & 10.50 & \\
\hline$\%$ Failed extubation & 8.00 & 26.30 & \\
\hline$\%$ Motor vehicle crash & 13.90 & 10.50 & \\
\hline$\%$ VDFR only & 15.70 & 5.30 & \\
\hline$\%$ Other & 50.40 & 47.40 & \\
\hline
\end{tabular}

Note:-VDRF indicates ventilator-dependent respiratory failure.

nificantly larger DPST on average, $6.2 \mathrm{~cm}$ versus $2.2 \mathrm{~cm}(P<$ $.001)$. The $95 \%$ CIs for BMI and DPST by extender use were the following: 27.4-29.4 (BMI, standard-length tube), 40.956.9 (BMI, extended-length tube), 2.0-2.3 (DPST, standard length tube), and 5.3-7.1 (DPST, extended-length tube).

The unadjusted OR per unit increase in DPST (centimeters) predicting proximal extended-length tube use was 5.1 (95\% CI, 2.9-9.1; $P<.001)$. After adjustment for arm position (final model), the OR per unit increase in DPST predicting proximal extended-length tube use was 8.1 (95\% CI, 3.618.2; $P<.001$ ). Those patients with a greater DPST had significantly higher odds of having a proximal extendedlength tube used. The interaction between DPST and arm position was checked but was not included in the final model. Instead, a logistic model of throat depth predicting proximal extended-length tube use was created, stratified by arm position. It was found that among those with a raised-arm position, the OR of extender use was 14.5 (95\% CI, 2.6-81.2), and among those with a lowered arm position, the OR was 5.5 (95\% CI, 2.4-12.4). With these models, the predicted probability of a proximal extended-length tracheotomy tube based on DPST was calculated. These data, for both the arms-up and arms-down positions, are graphically displayed in Figs 1 and 2.

The alternate model demonstrated that increasing BMI or having an otolaryngologist surgeon both had significantly higher ORs of a proximal extended-length tube being used (BMI: 1.2, 95\% CI, 1.1-1.3; ENT: 3.2, 95\% CI, 1.0-9.7). Neither the final model for BMI (adjusted for ENT) nor that for DPST (adjusted for arm position) displayed evidence of lack of fit by the Hosmer-Lemeshow test. Comparison of the 2 by using the pseudo- $R^{2}$ measure for model comparison purposes suggested that the DPST model was the better predictive model. 


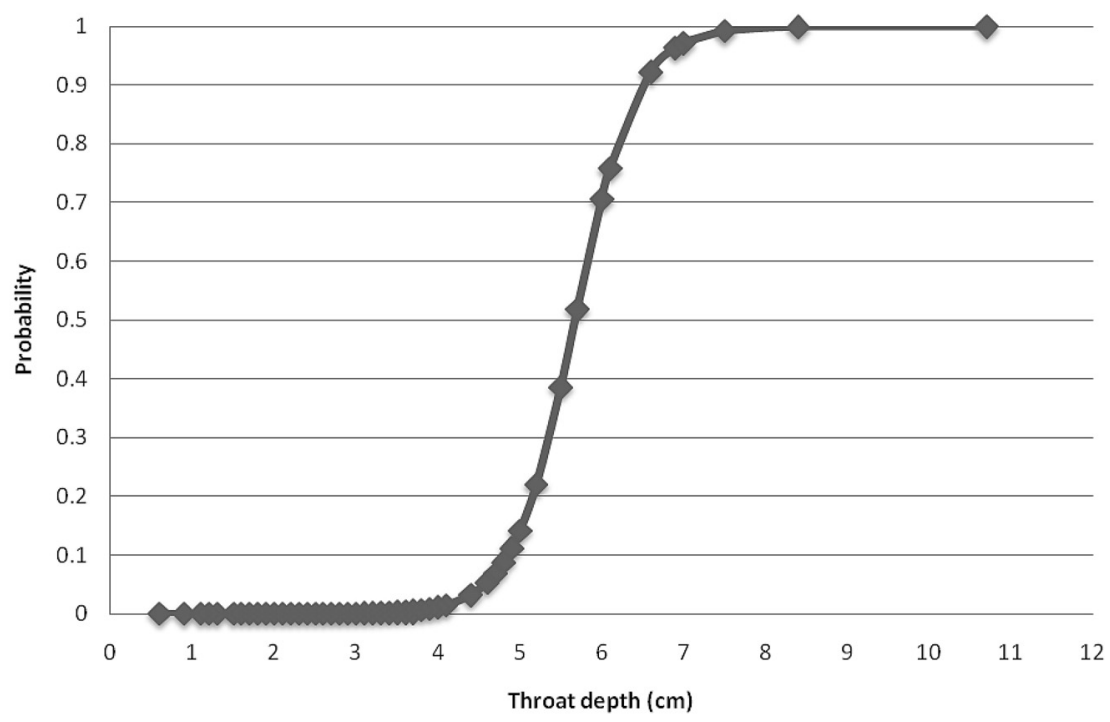

Fig 1. Predicted probabilities of proximal extended-length tube use by throat depth (arm-up position).

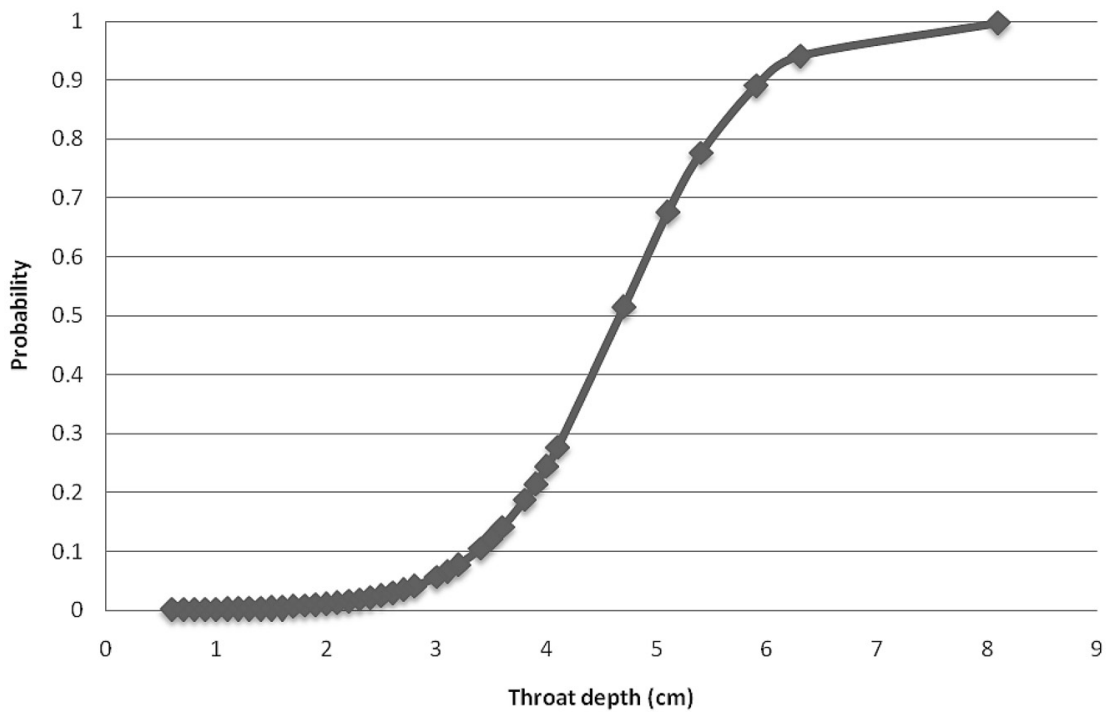

Fig 2. Predicted probabilities of proximal extended-length tube use by throat depth (arm-down position)

\section{Discussion}

Image-based measurement is common in many specialties; presurgical measurement for orthopedic implants and intravascular devices is considered standard of care. To our knowledge, however, this has not been applied to the placement of tracheotomy tubes. Here we sought to establish a baseline to determine if cross-sectional imaging could provide meaningful information to the preprocedural work-up of tracheotomy.

The use of CT has increased dramatically in recent years, and as such, many patients have preprocedural imaging evaluation that covers the expected anatomic area of tracheotomy tube placement. In our study, more than half of the patients (56\%) had imaging performed within 1 month before their procedure. Anecdotally, it was suggested in the patient's chart that an outside study was available for review in most of the remaining patients, and as such, most patients who present for tracheotomy probably have an available study for measurement. The aim of this study was not to encourage overuse of cross-sectional imaging or indeed to perform cross-sectional imaging for the purposes of obtaining this measurement. Instead, this illustrates the point that the information about the DPST is readily available in most cases.

The measurement of DPST on CT proved to be an easily performed technique that reliably predicted the use of a proximal extended-length tube at surgery. Stratified logistic regression models (Figs 1 and 2) indicate that above $3.9 \mathrm{~cm}$ in the arms-down position and above $5.0 \mathrm{~cm}$ in the arms-up position, there is a $20 \%$ probability of needing a proximal extended-length tube; above these depths, the probability rapidly increases. It is at these levels, therefore, that the use of a proximal extended-length tube should be entertained and made available during surgery.

The type of scan performed made considerable difference in the depth of anterior soft tissue. In the arms-down position (typically performed as a neck CT), the depth of anterior soft tissue was significantly less than that in the arms-up position (typically performed as a chest CT), though chest CT does not 
typically involve imaging the thyroid or tissues cranial to the thyroid gland. Knowledge of patient positioning is, therefore, important because application of the measurements differs (as above). The advantage, however, is that the use of any CT that covers the appropriate anatomy is possible.

Another objective measurement, BMI, also had a direct correlation with the choice of tracheotomy tube type. The predictive value is less than that of CT however. In the absence of imaging covering the appropriate anatomy, other metrics can be substituted, and performing a CT for the purposes of measurement is not necessary. Alternatively, a method of measuring the DPST by sonography has been recently described. ${ }^{6}$ Although sonography was not evaluated in our study, it would be expected that the results of measurement by sonography would be applicable to this model, depending on patient positioning. If a CT scan covering the appropriate anatomy is not available, CT should not be the imaging technique of choice if a measurement of DPST is desired before surgery. Sonography has multiple advantages over CT, including lack of ionizing radiation, portability, speed, and low cost.

This retrospective analysis has several drawbacks. Foremost, patient follow-up was extremely limited. Tracheotomy is often the last procedure to be performed before the patient leaves our facility for a long-term rehabilitation or nursing environment. Analysis was based on the recorded surgical note for the procedure, which may not have included a notation regarding the use of a proximal extended-length tube.

\section{Conclusions}

The DPST measurement on CT is an easily performed metric, which has significant correlation to the use of a proximal extended-length tracheotomy tube. This model should be verified and refined by prospective application. The use of CT specifically for the preoperative evaluation before tracheotomy is not endorsed because physical metrics (ie, BMI) or alternative imaging such as sonography have multiple benefits. However, with the increasing use of imaging, the CT data may be available for many patients and can be quickly and easily reviewed before surgery and have the potential to reduce the risk of early decannulation.

\section{References}

1. Goldenberg D, Bhatti N. Management of the impaired airway in the adult. In: Cummings CW, ed. Otolaryngology Head and Neck Surgery. 4th ed. Philadelphia: Mosby; 2005:2441-53

2. Solh AA, Jaafar W. A comparative study of the complications of surgical tracheostomy in morbidly obese critically ill patients. Critical Care 2007;11:R3

3. Byhahn C, Lischke V, Meininger D, et al. Peri-operative complications during percutaneous tracheostomy in obese patients. Anaesthesia 2005;60:12-15

4. De Leyn P, Bedert L, Delcroix M, et al. Tracheotomy: clinical review and guidelines. Eur J Cardiothorac Surg 2007;32:412-21. Epub 2007 Jun 27

5. Hess DR. Tracheostomy tubes and related appliances. Respir Care 2005;50: 497-510

6. Szeto C, Kost K, Hanley JA, et al. A simple method to predict pretracheal tissue thickness to prevent accidental decannulation in the obese. Otolaryngol Head Neck Surg 2010;143:223-29 